\title{
Revisiting preparation routes of SERS materials
}

\author{
A. A. Semenova ${ }^{1}$, I. A. Semenova ${ }^{2}$, A. P. Semenov ${ }^{2}$, E. A. Gudilina ${ }^{3}$, E. A. Goodilin ${ }^{1,4, *}$ \\ ${ }^{1}$ Lomonosov Moscow State University, Faculty of Materials Science, Lenin Hills, Moscow, 119991, Russia \\ ${ }^{2}$ Institute of Physical Materials Sciences of Siberian Branch of the Russian Academy of Sciences, \\ Buryatia Republic, Ulan-Ude, 670047, Russia \\ ${ }^{3}$ FSBI "N. N. Blokhin National Medical Research Center of Oncology" of the Ministry of Health \\ of the Russian Federation, Moscow, 115478, Russia \\ ${ }^{4}$ Kurnakov Institute of General and Inorganic Chemistry of the Russian Academy of Sciences, \\ Moscow, 119992, Russia \\ *goodilin@yandex.ru
}

PACS 61.46.Hk, 62.23.St, 68.35.bd

DOI 10.17586/2220-8054-2017-8-5-670-676

\begin{abstract}
For the first time, a systematic study of a background noise to signal ratio is given for various preparation histories of consolidated silver nanoparticles and artificially prepared nanostructures to rate the best and the worst routes of deposition of surface-enhanced Raman spectroscopy (SERS) active layers. It is shown that most of common preparation schemes face with a high intensity of extra peaks in the ca. 900-1100 and $1400-1700 \mathrm{~cm}^{-1}$ range as related to residual adsorbed / chemosorbed nitrate, nitrite ions and organic oxidation products of various pollutants formed in the course of $\mathrm{Ag}^{+}$redox reactions. Finally, Leopold-Lendl and the original USR (Ultrasonic Silver Rain) methods would be recommended for the highly sensitive SERS analysis of diluted solutions and impurities.
\end{abstract}

Keywords: SERS, silver, nanostructures, nanoparticles, preparation methods, background.

Received: 20 August 2017

Revised: 23 August 2017

\section{Introduction}

The Surface-enhanced Raman spectroscopy (SERS) method has become one of the most powerful and universal tool for modern analytics in pharmacology, ecology and potentially noninvasive biomedical diagnostics for screening and personal medicine [1-5]. Silver nanoparticles and nanostructured materials are a common choice for SERS measurements due to their broad plasmon resonance, high stability, facile fabrication methods and the largest enhancement for special molecules [3-5]. A complex morphology such as nanoflowers, nanorices, cubes, multipods and nanodendrites, mesocages is achieved by kinetically controlled, aggregation-based, heterogeneously seeded, template-directed growth, selective etching and colloidal system aging; the polyol technique allows one to synthesize silver nanoparticles of a wide variety of shapes and sizes [6-16]. Despite of all possible advantages, the nanoparticles themselves are likely to aggregate uncontrollably in solutions, making it difficult to reproduce SERS results $[5,6,11,14]$; nanoparticle sols exhibit limited plasmonic tunability as compared to a new class of nanomaterials - colloidosomes composed of a dielectric core and a concentric metal shells with hybridization of plasmon modes supported by an inner cavity and an outer surface of the nanoshell $[5,7,9,10]$. However, SERS still suffers a lack of reproducibility due to the masking of SERS signals by surfactants or by-products, gradients of electric fields near the nanoparticles and variations of molecule positions with respect to the nanoparticles; commonly used linkers, surfactants, reducing agents and their oxidized forms, halide ions can change the characteristic properties of nanoparticles because of their aging, recrystallization or random formation of aggregates [5,11-14]. Nanostructured substrates promise much better reproducibility and less toxicity promoting their SERS applications in biology and medicine, especially in highly sensitive lab-on-a-chip devices [5,11,13-15]. However, the coming wave of SERS development admits a challenge of development of highly sensitive and reproducible low cost nanomaterials vitally demanded for SERS. In this paper, a systematic study of a background noise to signal ratio is given for various preparation histories of consolidated silver nanoparticles to rate the routes of deposition of SERS active layers in the sake of practical development of SERS active materials for novel operating sensor devices.

\section{Experimental}

Silver nitrate $\left(\mathrm{AgNO}_{3}\right)$, sodium hydroxide $(\mathrm{NaOH})$, ammonium hydroxide $\left(\mathrm{NH}_{4} \mathrm{OH}, 30 \%\right)$, ascorbic acid, polyvinylpyrrolidone (PVP), sodium borohydride $\left(\mathrm{NaBH}_{4}\right)$, sodium citrate, hydrogen peroxide $\left(\mathrm{H}_{2} \mathrm{O}_{2}, 10 \%\right)$, hydrochloric acid $(\mathrm{HCl})$, Rhodamine $6 \mathrm{G}(\mathrm{Rh} 6 \mathrm{G})$ were of high purity grade. In all the experiments, ultra-pure 
water with resistivity ca. $18 \mathrm{M} \Omega \cdot \mathrm{cm}$ (Milli-Q, Millipore) was used. Several series of silver nanoparticles and nanostructures were prepared (Table 1) according to known (Series A-C, I) [1-3,6,7, 11-17] and new schemes (Series D-H, J).

TABLE 1. Series of experimental nanostructured silver samples and their preparation schemes

\begin{tabular}{|c|c|}
\hline Series & Preparation scheme \\
\hline Series A & Direct reduction of silver nitrate $(0.01-0.001 \mathrm{M})$ with ascorbic acid $(0.01-0.001 \mathrm{M})$ \\
\hline Series B & Direct reduction of silver nitrate $(0.01-0.001 \mathrm{M})$ with $\mathrm{NaBH}_{4}(0.01-0.001 \mathrm{M})$ \\
\hline Series C & Silver platelets preparation by grain selective regrowth of nuclei $[11,14]$ \\
\hline Series D & $\begin{array}{l}\text { Direct reduction of fresh water suspension of solid silver (I) oxide with an excess of } \\
\mathrm{H}_{2} \mathrm{O}_{2}(0.1-0.01 \mathrm{M})\end{array}$ \\
\hline Series E & Direct reduction of silver nitrate $(0.01-0.001 \mathrm{M})$ with water suspension of $\mathrm{SnCl}_{2}$ \\
\hline Series F & Reduction of diamminesilver hydroxide with hot microfiber cellulose suspension \\
\hline Series G & Reduction of diamminesilver hydroxide with hot graphene oxide suspension \\
\hline Series $\mathrm{H}$ & $\begin{array}{l}\text { Reduction of suspension of silver (I) chloride in hydrochloric acid }(0.01 \mathrm{M}) \text { with } \\
\text { metallic aluminum (hydrogen in situ) }\end{array}$ \\
\hline Series I & $\begin{array}{l}\text { The Leopold-Lendl method, reduction of silver nitrate with alkali solution of hydrox- } \\
\text { ylamine hydrochloride }[6,8,16]\end{array}$ \\
\hline Series J & Soft thermal pyrolysis of diamminesilver hydroxide $[5,12,15]$ \\
\hline
\end{tabular}

The samples of Series A and B were prepared by simple reduction of silver (I) nitrate in aqueous solution with commonly used organic and inorganic substances as noted in Table 1.

In accordance with our previous publications [11,14], highly pure water, silver (I) nitrate, sodium citrate, PVP, $\mathrm{NaBH}_{4}$, ascorbic acid were used as reagents to prepare silver nanoplates in the Series C (Table 1). Initially, silver (I) nitrate was reduced by $\mathrm{NaBH}_{4}$ in the presence of sodium citrate to form a seeding solution of spherical silver nanoparticles. In particular, $11 \mathrm{ml}$ aqueous solution of $0.11 \mathrm{mM} \mathrm{AgNO} 3$ and $2.05 \mathrm{mM}$ sodium citrate were mixed quickly, under vigorous stirring, with $0.3 \mathrm{ml}$ of freshly prepared $5 \mathrm{mM}$ aqueous solution of $\mathrm{NaBH}_{4}$. The stirring has been stopped in $10 \mathrm{~min}$. The yellow sol thus obtained was maintained for $5 \mathrm{hrs}$ at room temperature and then placed in a dark place at $4{ }^{\circ} \mathrm{C}$ for storage as a seeding solution. On the second stage, a mixture of aqueous solutions of $0.25 \mathrm{ml}$ of $5 \mathrm{mM} \mathrm{AgNO}_{3}, 0.75 \mathrm{ml}$ of $30 \mathrm{mM}$ sodium citrate, $0.75 \mathrm{ml}$ of $0.7 \mathrm{mM}$ (with respect to repeating fragment - chain) of PVP in $9.25 \mathrm{ml}$ of pure water was added to a series of aliquots of the seeding solution, namely $0.9,2.7,3.6,5.3,6.9,12.9,18.2,22.9,30.8,37.2,52.7$ vol. $\%$, followed by an addition of $6.25 \mathrm{ml}$ of $1 \mathrm{mM}$ of ascorbic acid. The solution color was found to start its changing immediately after ascorbic acid injection then it has become permanently stable after about $15 \mathrm{~min}$. Thus, prepared sols were purified by repeated centrifugation (19.900 rpm for $10 \mathrm{~min}$, Sartorius Sigma 3-30 K) followed by gentle dilution with pure water, after that they were stored at $4{ }^{\circ} \mathrm{C}$ in darkness.

In the Series D, silver (I) oxide prepared as in the Series C, was reduced to nanoparticles using hydrogen peroxide solutions while in the Series $\mathrm{E}$, a suspension of soft reducing species $\mathrm{SnCl}_{2}$ was used to get a composite with silver nanoparticles from silver nitrate solution (Table 1). In the Series F and G diamminesilver hydroxide solution was heated at $90-95{ }^{\circ} \mathrm{C}$ together with either suspension of microfiber cellulose or graphene oxide for about 30-60 min to deposit silver nanoparticles reduced by superficial groups of the substances onto the surface of cellulose of graphene (Table 1). In the Series H, silver chloride suspension was rapidly reduced by hydrogen "in situ" in the environment of hydrochloric acid, metallic aluminum was applied to generate hydrogen (Table 1). The Series I was exactly the silver colloid prepared by the classical Leopold and Lendl scheme [16] (Table 1).

In order to prepare silver ring nanostructures in the Series J, our original procedure $[5,12,15,17]$ was slightly modified (Table 1). SERS-active substrates containing nanostructured silver were prepared using spray pyrolysis deposition of aqueous diamminesilver hydroxide. Aqueous sodium hydroxide solution $(0.1 \mathrm{M})$ was added dropwise to a freshly prepared aqueous silver nitrate solution $(10 \mathrm{mM})$ until complete precipitation of a black-brown silver (I) oxide. Then, the prepared oxide was thoroughly washed with deionized water and dissolved in a twofold molar excess of a $10 \%$ aqueous ammonia solution (prepared from $30 \%$ ammonium hydroxide) to give a 
$0.0125 \mathrm{M}$ solution of a silver (I) complex; a higher concentration deteriorates the silver ring structure formation while superstoichiometric ammonia is needed to prevent $\mathrm{Ag}_{2} \mathrm{O}$ precipitation at the aerosol production stage. The obtained transparent silver complex solution was filtered through Millex-LCR syringe driven filter units (Millipore, $0.45 \mathrm{~mm}$ pores). In the ultrasonic silver rain (USR method) deposition process, this initial ammonia solution of silver (I) oxide was nebulized into mist and 1-5 $\mu$ m droplets were streamed onto "warm" $\left(270-290{ }^{\circ} \mathrm{C}\right)$ glasses.

The obtained materials were examined by XRD measurements using Rigaku D/MAX 2500 (Japan) with a rotating copper anode ( $\mathrm{CuK} \alpha$ irradiation, 5-90 ${ }^{\circ} 2 \theta$ range, $0.02^{\circ}$ step). Diffraction maxima were indexed using the PDF-2 database. UV-vis absorption spectra were recorded using the UV-vis spectrophotometer Lambda 950 (Perkin-Elmer) with an attached diffuse reflectance accessory. Measurements were in the spectral range 250$850 \mathrm{~nm}$ with a step scan of $1 \mathrm{~nm}$. Raman and SERS experiments were performed using InVia Raman microscope (Renishaw, UK) equipped with a $20 \mathrm{~mW} 514 \mathrm{~nm}$ argon laser and power neutral density filter (10\%). All the spectra were collected using $\times 50$ objective lens and $10 \mathrm{~s}$ of acquisition time. A silicon wafer was used for calibration.

\section{Results and discussion}

It is almost a common belief that formation of silver nanoparticles of a certain size by most of preparation methods guarantees SERS amplification of any substance [1-3]. Unfortunately, it is not as simple and certain as reported, and a lot of criteria should be satisfied prior achieving essential SERS effects $[5-15,17,18]$. It is also obvious that intense background peaks would be one of the most serious reasons to expect worsening analytical signals of SERS-based sensors. The data of Fig. 1 and Table 1 demonstrate that such drawbacks are unexpectedly common for many usual preparation schemes despite of the feelings one could expect after reading the literature; such unsatisfactory backgrounds are observed with no respect to application of either organic or inorganic reducing agents [1-3].

Silver itself has lattice vibrations and some special modes only in the range of $50-250 \mathrm{~cm}^{-1}$, these modes (especially the $230-235 \mathrm{~cm}^{-1}$ line) are clearly seen if silver is nanodispersed (Fig. 1) while all other peaks would not be related to metallic silver [19]. Thus the most expected reasons of the observed high background signals (Fig. 1) include the tremendous affinity of nanoparticle ensemble, possessing highly developed surface area values, with respect to the preparation byproducts. This is, in turn, connected with unsaturated superficial silver bonds, the existence of high zeta-potentials and a double layer for the nanoparticles in reacting solutions, moreover formation of aggregated nanoparticle structures results in capillary absorption and all of these factors lead to blocking the nanoparticle surface with the chemisorbed molecules of reactants, product impurities or surfactants applied for the synthesis. This finally means that these impurities should provide a certain number of Raman peaks strongly enhanced in intensities due to the SERS effect, thus creating a noisy background including an important spectral range of $1000-1700 \mathrm{~cm}^{-1}$ and the fingerprint area of $2500-3000 \mathrm{~cm}^{-1}$ (Fig. 1, Table 2).

As evident from Fig. 1 and Table 2, the most widely used reductants like ascorbic acid, $\mathrm{NaBH}_{4}$ and hydrogen peroxide give a risk of a noisy background. In the case of hydrogen peroxide, the background appears because of substances stabilizing $\mathrm{H}_{2} \mathrm{O}_{2}$ such as polyphosphates, salicylic acid, etc. contributing their own modes to the spectra. The same reason leads to the peaks in the case of ascorbic acid which is one of the carbon acids with typical vibrations of the $-\mathrm{CO}_{2}$ fragment, some other peaks could be given by its oxidation products chemisorbed onto the silver surface. $\mathrm{NaBH}_{4}$ is degradable in surrounding moisture and $\mathrm{CO}_{2}$ atmosphere giving carbonate-like peaks and some peaks near $3000 \mathrm{~cm}^{-1}$ due to the precipitation of $\mathrm{B}_{2} \mathrm{O}_{3} \cdot x \mathrm{H}_{2} \mathrm{O}$ with internal hydrogen bonds; peaks near $1000 \mathrm{~cm}^{-1}$ would be related to chemisorbed nitrates. The least intense background is demonstrated by the nanostructured silver prepared by reduction with hydroxylamine hydrochloride in ammonia environment of silver chloride purified by repeated washing and centrifugation after its precipitation with an excess of hydrochloric acid from silver nitrate. Such a procedure gets rid of nitrates and other impurity ions; the inorganic reductant forms gaseous nitrogen which could not disturb SERS spectra measurements. Small peaks at $1300-1550 \mathrm{~cm}^{-1}$ are most probably related to amide bonds forming between silver and residual ammonia. The peaks near $3000 \mathrm{~cm}^{-1}$ seem to be attributed to hydrogen bonds in water absorbed with aggregated structure of silver nanoparticles [20]. For example, an application of hydroxyethylcellulose, swelling in both hydrophilic and hydrophobic environment, combined with the Series I samples, gives a small worsening of the background signal mostly because of hydrogen bonds associated with residual water. Microcellulose carriers are cheap and easily available materials with a small Raman cross-section. Precipitation of silver nanoparticles by a direct reaction of cellulose suspension with a hot $\left(80-90{ }^{\circ} \mathrm{C}\right)$ 0.001-0.01 M solution of diamminesilver hydroxide for 10-15 min gives latter a dry material with a high absorption ability and a low background signal (Table 2).

Finally, thermal decomposition of diamminesilver hydroxide solutions (see the Experimental part) allows preparing nanostructured silver materials with a uniformly low background signal not exceeding 3-5\% of analyte 


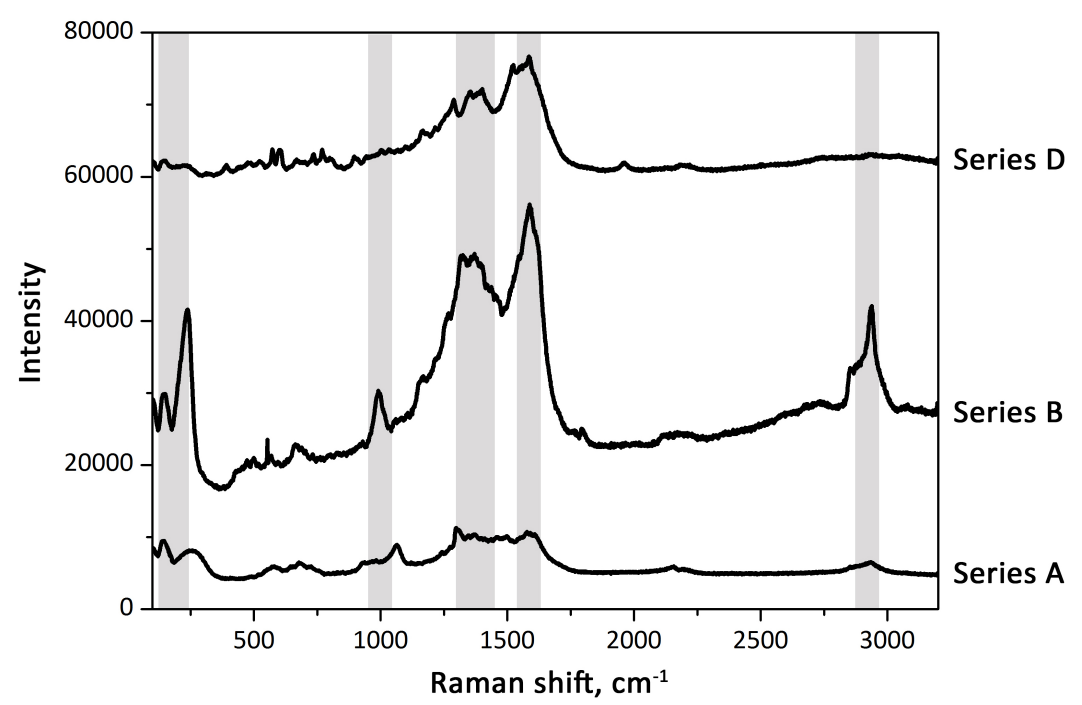

(a)

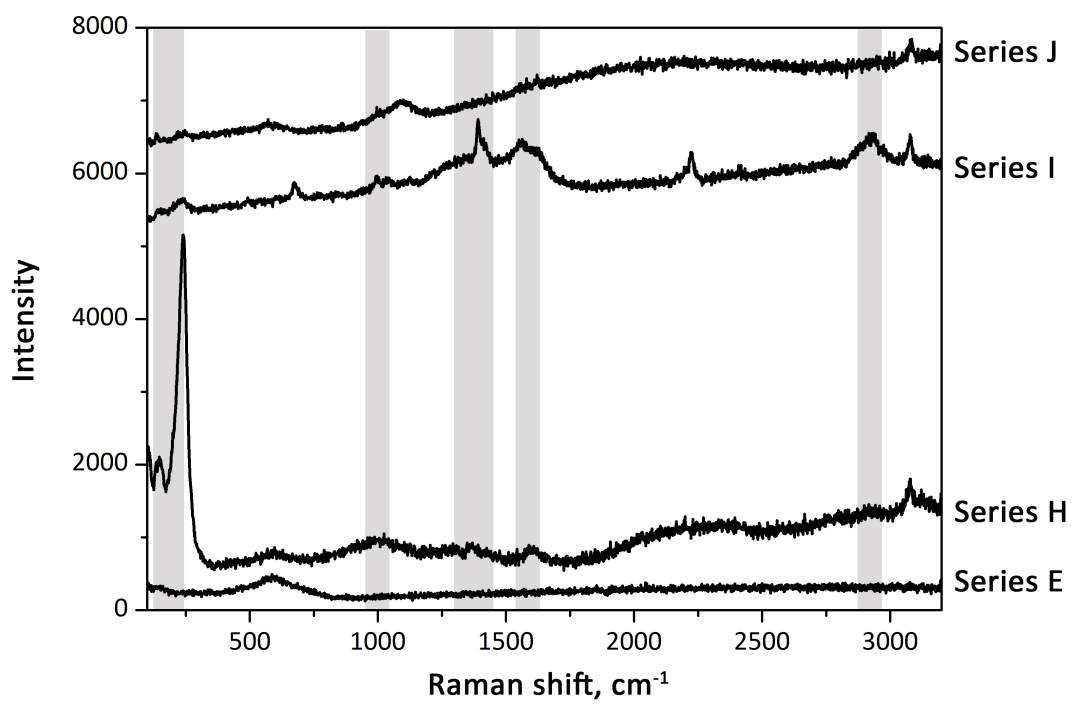

(b)

FIG. 1. Typical raw background signals of dried powdered silver nanoparticles with a different preparation history: (a) series with a large backgrounds, (b) series with smooth and low background signal

signals in the whole working range of our spectrometer. The latter becomes possible because of a combination of highly nonequilibrium preparation conditions guaranteeing a small size for the formed nanoparticles and their aggregates and also high enough temperatures prevent the physical adsorption and chemisorption of byproducts. Moreover, pyrolytic deposition is a scalable method suitable for preparation of either nanoparticles of a complex shape or planar nanostructures $[12,13,15]$. All of such nanostructures reveal the large number of "hot spots" and are free of nonvolatile impurities since there are no special reductants or surfactants applied in the preparation route of these original materials. Fig. 2 evidently demonstrates that the nanostructured samples with selected preparation histories diminishing background signals show remarkable enhancement coefficients and seem to be effective materials for the development of new SERS sensors.

From a chemical perspective, thermal treatment of ultrasonic mists of the diamminesilver hydroxide complex provides irreversible decomposition and solvent evaporation. The products of this transformation include silver nanostructured particles, water, ammonia and oxygen. Quenching of such products yields nanostructured particles and their aggregates formed in a shock manner, since the whole transformation occurs for few seconds at high temperatures $[12,13,15]$. High-temperature silver microspheres of about $100 \mathrm{~nm}$ in size are typical for aerosol spray pyrolysis because of solvent evaporation from mist droplets and spontaneous formation of silver nuclei 
TABLE 2. Functional properties of samples with different preparation schemes

\begin{tabular}{|c|c|c|}
\hline $\begin{array}{l}\text { Series } \\
\text { of samples }\end{array}$ & $\begin{array}{l}\text { Background signal / observed Raman background peaks } \\
\text { for pure consolidated sample of nanostructure silver } \\
\text { nanoparticles or nanostructures }\end{array}$ & $\begin{array}{l}\text { SERS activity with } \\
\text { respect to Rhodamine } \\
6 \mathrm{G}(\mathrm{Rh} 6 \mathrm{G})\end{array}$ \\
\hline A & Strong, peaks at $150,240,1300-1400,1600,2900 \mathrm{~cm}^{-1}$ & Average, $10^{-6} \mathrm{M}$ \\
\hline B & Strong, peaks at $150,240,1300-1400,1600,2900 \mathrm{~cm}^{-1}$ & Average, $10^{-6} \mathrm{M}$ \\
\hline $\mathrm{C}$ & $\begin{array}{l}\text { Average, peaks at } 150,240, \text { a wide double peak at } 1300 \\
1600,2900 \mathrm{~cm}^{-1}\end{array}$ & Average, $10^{-6} \mathrm{M}$ \\
\hline $\mathrm{D}$ & Strong, peaks at $150,240,1300-1400,1600,2900 \mathrm{~cm}^{-1}$ & Average, $10^{-6} \mathrm{M}$ \\
\hline $\mathrm{E}$ & Weak, peaks at $150,240 \mathrm{~cm}^{-1}$ & Small, $10^{-4} \mathrm{M}$ \\
\hline $\mathrm{F}$ & Average, peaks at $1300-1400,1600,2900 \mathrm{~cm}^{-1}$ & Good, $10^{-8} \mathrm{M}$ \\
\hline G & Average, peaks at $1400,1600(\mathrm{D}, \mathrm{G}$ modes $) \mathrm{cm}^{-1}$ & Average, $10^{-6} \mathrm{M}$ \\
\hline $\mathrm{H}$ & No obstacles from background & $\begin{array}{l}\text { No activity, } \\
\text { bulk metallic silver }\end{array}$ \\
\hline I & Weak, peaks at $150,240,1100,2900 \mathrm{~cm}^{-1}$ & High, $10^{-8}-10^{-10} \mathrm{M}$ \\
\hline $\mathrm{J}$ & No obstacles from background & High, $10^{-8}-10^{-10} \mathrm{M}$ \\
\hline
\end{tabular}

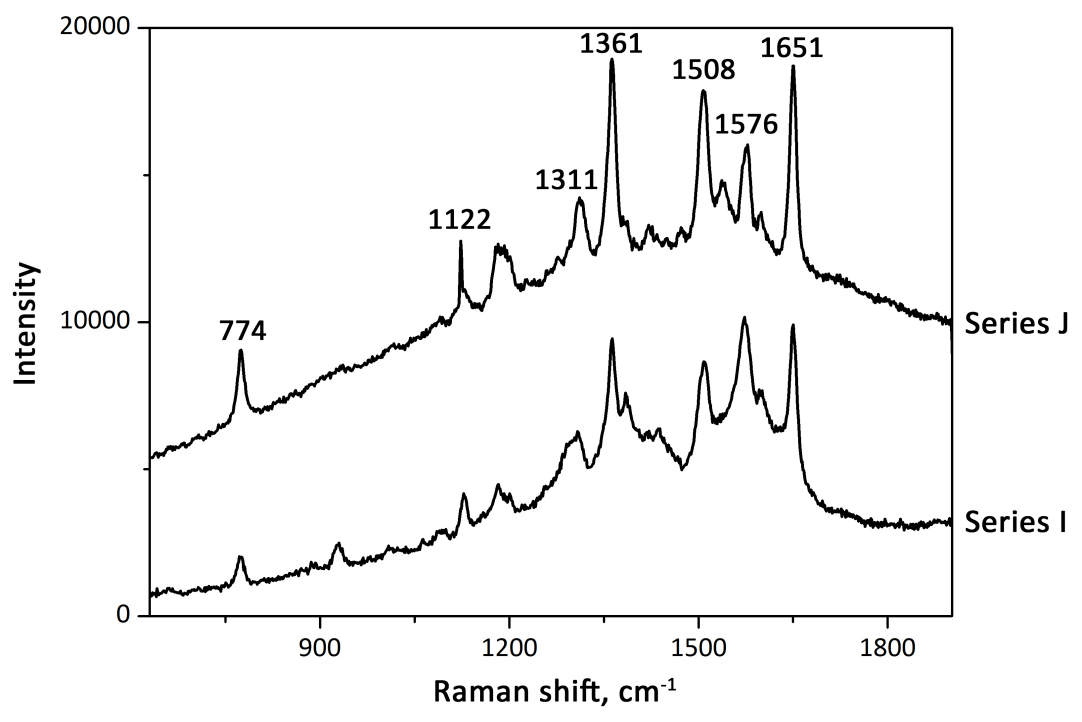

FIG. 2. Typical SERS spectra of model analyte (Rh6G dye) in different concentrations recorded using silver nanostructures: $1 \%$ of laser power, $10 \mathrm{~s}$ of acquisition time, $10^{-7} \mathrm{M}$ Rh6G (Series $\mathrm{J}), 5 \%$ of laser power, $10 \mathrm{~s}$ recording, $10^{-8} \mathrm{M}$ Rh6G (Series I)

joining into a porous "orange skin" layer. Polyhedral aggregates formed could be a consequence of primary silver (I) oxide formation within the mist droplets prior its decomposition. In the case of application of warm substrates (see the Experimental part), silver ring structures caused by the known "coffee ring" effect are formed at moderate temperatures of $200-250{ }^{\circ} \mathrm{C}$ due to the deposition of the mist droplets onto a preheated substrate (Fig. 3). The coatings consist of overlapping silver rings of a complex morphology originated from decomposition of micron-sized droplets of ultrasonic mist of silver (I) solution. This resembles a rain making rings on a flat dusty surface hence we call it UltraSonic Silver Rain (USR method). Usually, silver deposition gives intersecting circles of $30-100 \mu \mathrm{m}$ in diameter. This value is several times larger than the expected size of $1-10 \mu \mathrm{m}$ of the falling mist droplets as occurred because the liquid from the droplets spreads laterally over the substrate. Solvent evaporation increases concentration of the silver complex and then metallic silver resides on rims of the spreading circles producing walls of silver craters. The thickness of walls lies typically in a range of $1-3 \mu \mathrm{m}$ while the 
residual part is covered with sparse silver nanoclusters gradually increasing in their sizes from 10-20 nm in the centers of circles to about $100 \mathrm{~nm}$ in the wall vicinities. The nanoclusters increase the role of capillary forces and, afterwards, more droplets stack onto the surface, boil, decompose; an increase of sputtering time leads to rough and porous layers. This deposition mode has several advantages in terms of manufacturing SERS-active structures for promising applications: silver micro- and nanostructures are immobilized onto a substrate and form a rather stable metallic film on cheap materials like glass; there is no need to filter or separate nanoparticles; the nanostructure consists of porous silver sponge only; the temperatures are high enough for the one-stage formation of metallic silver nanostructured layer. The negligible background of the USR substrates allowed to use small laser power that prevents photoinduced damage of biological objects and ensures repeated measurements of SERS even from the same point $[7,15]$. Thus, this preparation technique is the method of choice for preparation of functional components for new SERS devices.

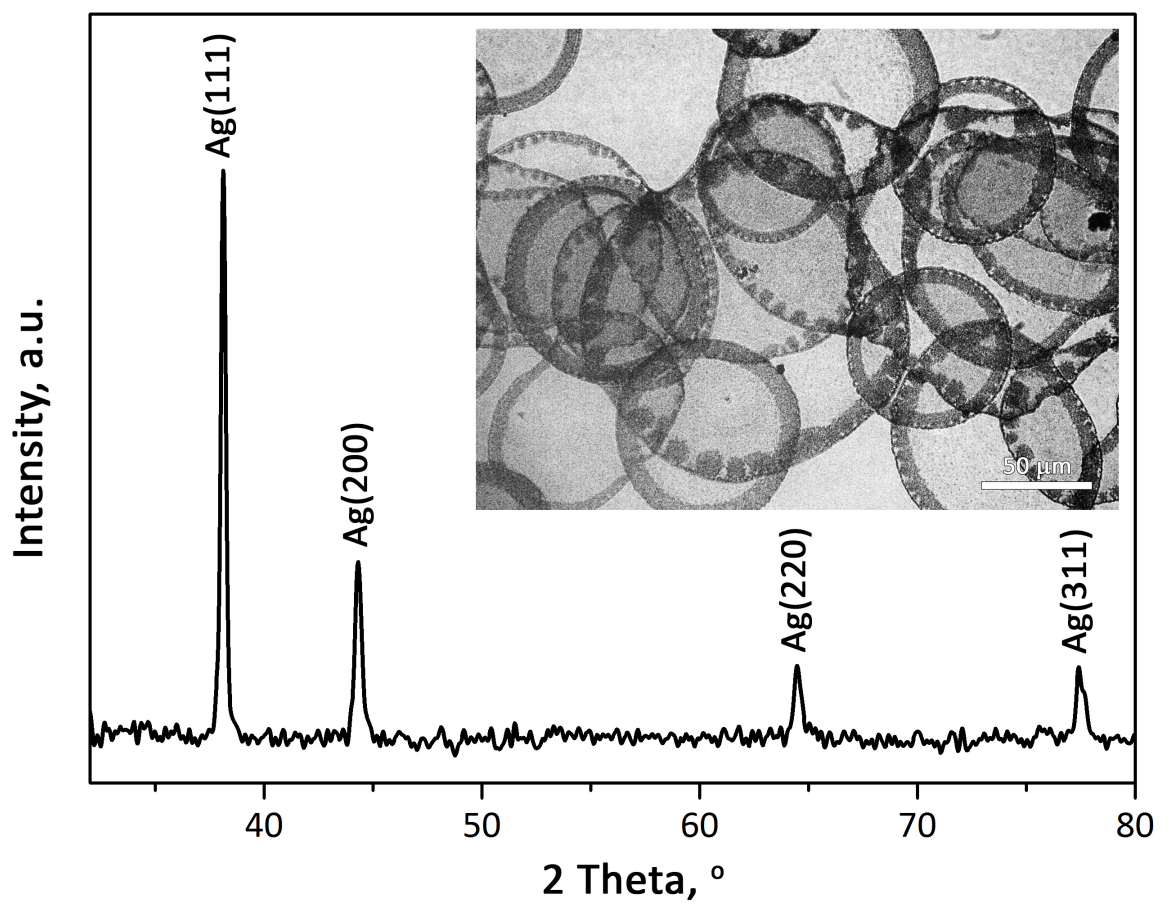

FIG. 3. Microstructural features of SERS-active samples with the pyrolysis preparation history (Series J): typical XRD data of the chemically coated silver substrates and transmission optical images of the ring structures (inset)

\section{Conclusions}

A systematic study of a background noise to signal ratio for various preparation histories of consolidated silver nanoparticles and artificially prepared nanostructures allowed us to rate the best and the worst routes of deposition for SERS active layers. It is shown that most of common preparation schemes face a high intensity of parasitic unidentified peaks in the ca. $900-1100$ and $1400-1700 \mathrm{~cm}^{-1}$ regions as related to residual adsorbed/chemisorbed nitrate, nitrite ions and organic oxidation products of various pollutants formed in the course of $\mathrm{Ag}^{+}$redox reactions. Finally, the Leopold-Lendl route and the original USR spray deposition method would be recommended for the highly sensitive SERS analysis of dilute solutions and impurities. The USR synthesis seems to be the method of choice for preparing functional components for new SERS devices.

\section{Acknowledgements}

This research is supported by the Grant of the President of Russian Federation for the governmental support of young scientists (\#MK-1146.2017.3). 


\section{References}

[1] Alvarez-Puebla R.A., Liz-Marzan L.M. SERS-based diagnosis and biodetection. Small, 2010, 6 (5), P. 604-610.

[2] Sharma B., Frontiera R.R., et al. SERS: Materials, applications, and the future. Mater. Today, 2012 , 15 (1-2), P. 16-25.

[3] Yuan Y., Panwar N., et al. SERS-based ultrasensitive sensing platform: An insight into design and practical applications. Coord. Chem. Rev., 2017, 337, P. 1-33.

[4] Tretyakov Y.D., Goodilin E.A. Chemical principles of the metall-oxide superconductors preparation. Uspekhi khimii, 2000 , 69 (1), P. 3-40.

[5] Semenova A.A., Semenov A.P., et al. Nanostructured silver materials for noninvasive medical diagnostics by surface-enhanced Raman spectroscopy (focus article). Mendeleev Communications, 2016, 26 (3), P. 177-186.

[6] Brazhe N.A., Parshina E.Y., et al. Tuning SERS for living erythrocytes: Focus on nanoparticle size and plasmon resonance position. Journal of Raman Spectroscopy, 2013, 44 (5), P. 686-694.

[7] Brazhe N.A., Evlyukhin A.B., et al. Probing cytochrome c in living mitochondria with surface-enhanced Raman spectroscopy. Scientific reports, 2015, 5, 13793

[8] Parshina E.Y., Sarycheva A.S., et al. Combined Raman and atomic force microscopy study of hemoglobin distribution inside erythrocytes and nanoparticle localization on the erythrocyte surface. Laser Physics Letters, 2013, 10 (7), 075607(6 pp.).

[9] Sarycheva A.S., Brazhe N.A., et al. New nanocomposites for SERS studies of living cells and mitochondria. Journal of Materials Chemistry B, 2016, 3 (4), P. 539-546.

[10] Sarycheva A.S., Ivanov V.K., et al. Microbead silica decorated with polyhedral silver nanoparticles as a versatile component of sacrificial gel films for SERS applications. RSC advances, 2015, 5 (110), P. 90335-90342.

[11] Semenova A.A., Goodilin E.A. Surface enhanced Raman spectroscopy substrates with advanced spectral sensitivity prepared from five years old silver nanoplatelets. Functional materials letters, 2016, 9 (6), 1642003(4 pp.).

[12] Semenova A.A., Ivanov V.K., Savilov S.V., Goodilin E.A. Unusual silver nanostructures prepared by aerosol spray pyrolysis. Cryst. Eng. Comm., 2013, 15 (39), P. 7863-7871.

[13] Semenova A.A., Brazhe N.A., et al. Aqueous diamminesilver hydroxide as a precursor of pure silver nanoparticles for SERS probing of living erythrocytes. Plasmonics, 2014, 9 (2), P. 227-235.

[14] Semenova A.A., Brazhe N.A., et al. A new route of SERS analysis of intact erythrocytes using polydisperse silver nanoplatelets on biocompatible scaffolds. RSC advances, 2016, 6 (88), P. 85156-85164.

[15] Semenova A.A., Goodilin E.A., et al. Planar SERS nanostructures with stochastic silver ring morphology for biosensor chips. Journal of Materials Chemistry, 2012, 22 (47), P. 24530-24544.

[16] Leopold N., Lendl B. A new method for fast preparation of highly surface-enhanced Raman scattering (SERS) active silver colloids at room temperature by reduction of silver nitrate with hydroxylamine hydrochloride. Journal of Physical Chemistry B, 2003, 107 (24), P. 5723-5727.

[17] Semenova A.A. Silver based nanostructured materials for biomedical diagnostics by Surface-enhanced Raman spectroscopy, PhD thesis, Moscow, 2012, 199 pp.

[18] Sidorov A.V. Chemically modified nanocomposites of silver for Surface-enhanced Raman spectroscopy of raw oil products, PhD thesis, Moscow, 2016, 220 pp.

[19] Kurbatova N.V., Galyautdinov M.F., et al. Low-temperature Raman spectroscopy of copper and silver nanoparticles ion-synthesized in a silica glass and subjected to laser annealing. Physics of the Solid State, 2010, 52 (6), P. 1255-1259.

[20] Kochkina N.E., Skobeleva O.A., Khokhlova Yu.V. Synthesis of silver nanoparticles in DMSO solutions of starch: a comparative investigation of native and soluble starches. Nanosystems: Physics, chemistry, mathematics, 2015, 6 (3), P. $405-411$. 